\title{
Oral Administration of Geranylgeraniol Rescues Denervation- induced Muscle Atrophy via Suppression of Atrogin-1
}

\author{
AKI MIYAWAKI ${ }^{1,2}$, THIRA ROJASAWASTHIEN ${ }^{1}$, SUZURO HITOMI $^{3}$, YOSHINORI AOKI ${ }^{4}$, \\ MARIKO URATA ${ }^{1}$, ASAKO INOUE ${ }^{1}$, TAKUMA MATSUBARA ${ }^{1}$, KAZUMASA MORIKAWA ${ }^{5}$, \\ MANABU HABU ${ }^{2}$, KAZUHIRO TOMINAGA ${ }^{2}$ and SHOICHIRO KOKABU ${ }^{1}$ \\ ${ }^{1}$ Division of Molecular Signaling and Biochemistry, Department of Health Improvement, \\ Kyushu Dental University, Kitakyushu, Japan; \\ ${ }^{2}$ Division of Oral and Maxillofacial Surgery, Department of Science and Physical Functions, \\ Kyushu Dental University, Kitakyushu, Japan; \\ ${ }^{3}$ Department of Physiology, Nihon University School of Dentistry, Tokyo, Japan; \\ ${ }^{4}$ Foods and Nutrition Science Div. Mitsubishi-Chemical Foods Corporation, Tokyo, Japan; \\ ${ }^{5}$ Division of Pediatric and Special Care Dentistry, Department of Developmental \\ Oral Health Science, School of Dentistry, Iwate Medical University, Morioka, Japan
}

\begin{abstract}
Background/Aim: Geranylgeraniol (GGOH), a C20 isoprenoid naturally occurs in several foods. We previously reported that GGOH treatment reduced the expression levels of Atrogin-1 which is involved in skeletal muscle degradation and stimulates the myogenic differentiation of $\mathrm{C} 2 \mathrm{C} 12$ myoblasts. However, the effect of GGOH supplementation on skeletal muscle metabolism in vivo is unknown. Materials and Methods: Skeletal muscle atrophy was induced by denervation. The expression levels of Atrogin-1 were assessed by western blotting or real time PCR. Results: Intraoral administration of $G G O H$ reduced the decrease in the cross-sectional area of muscle fibers and also suppressed the expression levels of Atrogin-1 in denervation induced muscle atrophy. Also, GGOH treatment suppressed the expression of Atrogin-1 and the decrease in skeletal muscle fiber size by glucocorticoid in vitro. Conclusion: Intraoral administration of GGOH rescues denervation-induced muscle atrophy via suppression of Atrogin-1.
\end{abstract}

Sarcopenia is an age-related decline in muscle strength and mass (1). Decreased muscle mass is often accompanied by increased insulin resistance. Sarcopenia has also various

This article is freely accessible online.

Correspondence to: Shoichiro Kokabu, DDS, Ph.D., 2-6-1 Manazuru, Kokurakita-ku, Kitakyushu, 803-8580, Japan. Tel: +81 932851131, Fax: +81932856000, e-mail: r14kokabu@fa.kyudent.ac.jp

Key Words: Geranylgeraniol, skeletal muscle atrophy, Atrogin-1. adverse consequences including poor quality of life, dependency, use of hospital services, institutionalization, and ultimately mortality (2). With the worldwide increase in the aging of the society there is an urgent need for strategies that will improve skeletal muscle function and mass in aging adults.

In addition to sarcopenia, skeletal muscle degradation also occurs in response to food deprivation, cancer cachexia, uremia, diabetes, cardiac failure, sepsis and muscle disuse $(3,4)$. Atrogin-1/MAFbx, a ubiquitin protein ligase, is involved in skeletal muscle degradation $(5,6)$. Atrogin- 1 is induced at an early stage of the atrophy process and increased atrogin- 1 expression is related with a reduction in muscle mass (5). Mice lacking atrogin-1 are resistant to skeletal muscle atrophy following denervation (6). Therefore, the induction of atrogin- 1 plays an important role in skeletal muscle atrophy. However, mechanisms by which Atrogin-1 expression is regulated are not fully understood.

Geranylgeraniol $(\mathrm{GGOH})$ is a terpenoid and polyprenoid occurring naturally in many plants and common foods such as rice. The United States Food and Drug Administration classifies GGOH as a Generally Recognized as Safe (GRAS) compound (7). In animals, GGOH occurs as a metabolite within the mevalonate pathway and as a precursor to geranylgeranylpyrophosphate (GGPP). Two phosphorylation events convert GGOH into the pyrophosphate moiety (8). GGPP induces protein geranylgeranylation, a requirement for plasma membrane localization of some proteins, particularly the small GTP-binding proteins Ras, Rho, Rac and Rap (9). Statins interact with the mevalonate pathway and are used for inhibiting cholesterol synthesis. Statins have few adverse side effects but can also cause muscle pain, myoblast damage, and rhabdomyolysis (10). Cao P et al., reported that 
A Schedule of $\mathrm{GGOH}$ administration

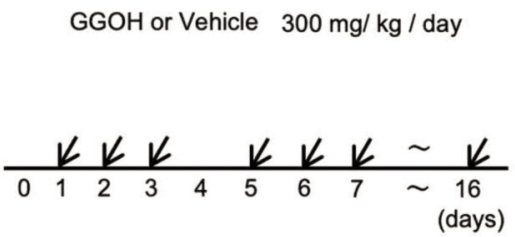

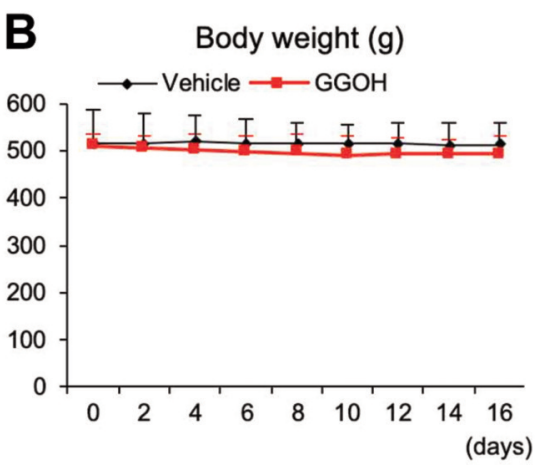

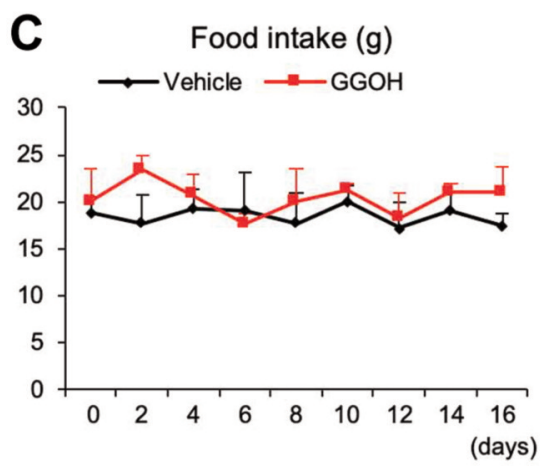

Figure 1. Optimization of administration schedules and dose of GGOH. Schematic of the GGOH administration schedule. Three hundred mg/kg $G G O H$ or control canola oil were administered intraorally once a day for 16 days with a rest day every fourth day (A). Body weight (B) and food intake $(C)$ were measured once a day. Data are mean $\pm S D(n=8)$.

GGOH treatment reduces expression levels of atrogin-1 as well as muscle damage induced by statins in vitro (11). We also previously reported that GGOH treatment reduced the expression levels of Atrogin-1 and moreover, dramatically stimulated myogenic differentiation of $\mathrm{C} 2 \mathrm{C} 12$ myoblasts (12). However, the effect of GGOH supplementation on skeletal muscle metabolism in vivo is completely unknown.

In present study, we demonstrated that oral administration of GGOH rescued the denervation-induced decrease in fiber size of murine skeletal muscle via decreasing the expression of Atrogin-1.

\section{Materials and Methods}

Administration of GGOH. Ten-week-old male Wister rats were purchased from Kyudo (Saga, Japan). $300 \mathrm{mg} / \mathrm{kg} \mathrm{GGOH}$ were administered intraorally using a disposable animal feeding needle (Fuchigami, Kyoto, Japan) once a day for 16 days with a rest day every fourth day. GGOH was kindly provided as geranylgeraniol$50^{\circledR}$ by Mitsubishi-chemical foods corporation (Tokyo, Japan). Geranylgeraniol- $50^{\circledR}$ is extracted from the seeds of annatto and mixed with canola oil. Geranylgeraniol- $50{ }^{\circledR}$ is prepared to contain 50\% GGOH (approval number: 17-003).

Muscle regeneration experiments. C57BL/6J mice were purchased from CLEA Japan Inc. (Tokyo, Japan). The tibial anterior muscle (TA) of 10-week-old male C57BL/6 mice was injected with $50 \mu \mathrm{l}$ of $10 \mu \mathrm{M}$ cardiotoxin (Sigma Aldrich Chemicals, St. Louis, MO, USA) using a 30-G syringe. Muscles were removed 14 days later and immediately frozen in isopentane cooled in liquid nitrogen. This experiment was performed in accordance with the guidelines of the experimental animal care and use committee of Kyushu Dental University (approval number: 17-003).

Muscle atrophy experiments. In anesthetized 7- week-old mice, the sciatic nerve of the right leg was cut and a $5 \mathrm{~mm}$ piece was excised. The left leg remained innervated and was used as a control (13). Gastrocnemius (GA) muscles were removed 6 days later, immediately frozen in isopentane cooled in liquid nitrogen, or prepared for RNA extraction. This experiment was performed in accordance with the guidelines of the experimental animal care and use committee of Kyushu Dental University (approval number: 17-004).

Histological analysis. Freshly dissected TA or GA muscles in OCT compound (Sakura Finetek Japan Co., Ltd, Tokyo, Japan) were flash frozen with liquid nitrogen or fixed immediately with $2 \%$ formaldehyde. $10 \mu \mathrm{m}$ sections were prepared using a cryostat (14). Sections were stained with hematoxylin and eosin (H\&E). 10x magnification images of the sections were acquired with a BZ-II Analyzer (KEYENCE, Osaka, Japan). Histomorphometric measurements were obtained with ImageJ (National Institute for Health, Bethesda, MD, USA). Muscle fibers for measurements included an intact, distinct cell membrane without significant signs of folding or distortion. Oblique sections with elongated fibers were excluded. Image analyses were performed by two authors (A.M. and S.K.)

Cell culture, and skeletal muscle atrophy in vitro. $\mathrm{C} 2 \mathrm{C} 12$ cells were purchased from American Type Culture Collection (Manassas, VA, USA). C2C12 cells were maintained as previously described (14). Well differentiated $\mathrm{C} 2 \mathrm{C} 12$ cells were cultured in the presence with 0,10 , or $50 \mu \mathrm{M} \mathrm{GGOH}$ (Sigma Aldrich Chemicals, St. Louis, MO, USA) along with or without $1 \mu \mathrm{M}$ dexamethasone (WAKO, Osaka, Japan) for 2 days. Dexamethasone was used to induce muscle atrophy (15). Skeletal muscle differentiation of $\mathrm{C} 2 \mathrm{C} 12$ cells was induced by culturing with medium supplemented with $2 \%$ horse serum for 4-8 days (14).

RNA isolation and quantitative real-time PCR ( $q P C R)$. Total RNA was isolated with FastGeneTM RNA Basic Kit (Nippon Genetics, Tokyo, Japan). cDNA was synthesized with High Capacity cDNA Reverse Transcription Kit (Applied Biosystems, ThermoFisher Scientific, Waltham, MA, USA). qPCR was performed using PowerUp SYBR Green Master Mix (ThermoFisher Scientific, Waltham, MA, USA) and a QuantStudio 3 Real-Time PCR System (ThermoFisher Scientific). Values were normalized to TATA box binding protein ( $T b p)$ using the $2^{\text {-DDCt }}$ method (16). The following primers were used for qPCR analyses: qPCR for murine Atrogin-1 (primer sequences: forward, agtgaggaccggctactgtg; reverse, gatcaaacgcttgcgaatct), murine Myogenin (primer sequences: 

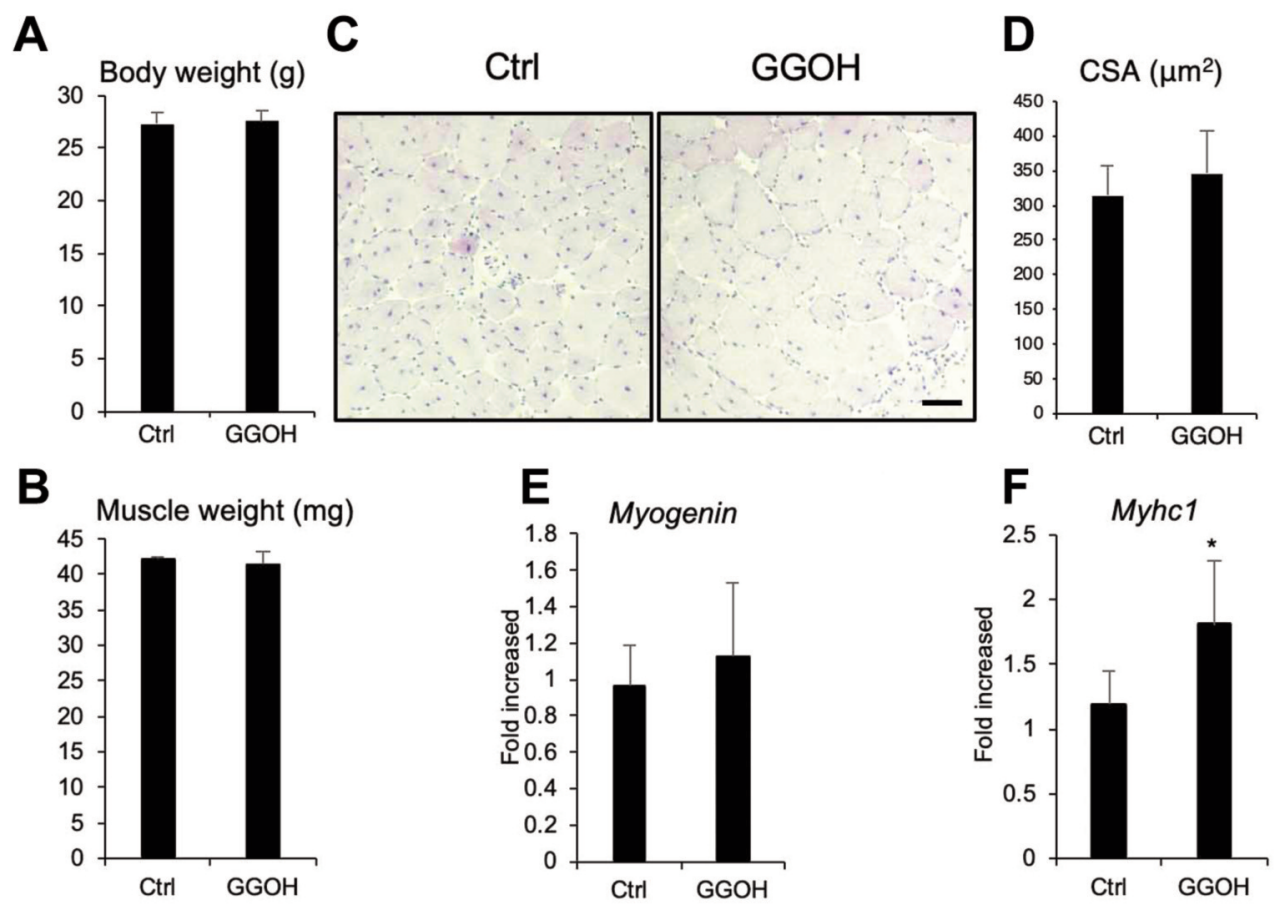

Figure 2. GGOH does not affect skeletal muscle regeneration induced by CTX injection (A-D) The effects of intra oral GGOH administration on total body weight (A), CTX injected tibial anterior muscle wet weight (B), and muscle cross sectional area (CSA) (C). CSA was quantified (D). Representative images are shown. Scale $=100 \mu \mathrm{m}(C)$. The mRNA levels of Myogenin (E) or Myosin heavy chain $1(M y h c 1)(F)$ from the TA muscle of mice administered GGOH or not were assessed by quantitative real-time PCR. Data are mean $\pm S D(n=6)$. * $p<0.05$, versus control (Ctrl).

forward, ccttgctcagctcctca; reverse, tgggagttgcattcactgg), murine Myhcl (primer sequences: forward, aatcaaaggtcaaggcctacaa; reverse, gaatttggccaggttgacat), and murine $T b p$ (primer sequences: forward, ggcggtttggctaggttt; reverse, gggttatcttcacacaccatga).

Immunocytochemistry. $\mathrm{C} 2 \mathrm{C} 12$ cells were incubated with anti-myhc mouse monoclonal antibody (MF20, R \& D Systems, Minneapolis, $\mathrm{MN}$, USA) for $1 \mathrm{~h}$ at room temperature following by blocking/permeabilization with phosphate-buffered saline containing $0.3 \%$ Triton $\mathrm{X} 100$ and $5 \%$ goat serum for $30 \mathrm{~min}$ at room temperature. The target proteins were visualized using an Alexa 488-conjugated secondary antibody (Invitrogen, Carlsbad, CA, USA) and imaged with a ABZ-9000 (Keyence, Tokyo, Japan) microscope. The circumference of each myosin heavy chain positive fiber was outlined using the ImageJ software (National Institute for Health) to calculate width of myofibers.

Western blot analysis. Rabbit Anti-Fbx32 (Atrogin-1) antibody (ab168372) was used for Western blot analysis, and HRP conjugated anti-GAPDH mouse monoclonal antibody (Proteintech, Chicago, IL, USA). The target proteins were detected using anti-rabbit IgG antibody conjugated with a horseradish peroxidase (Cell signaling, Beverly, MA, USA) and visualized by using ImmunoStar LD (WAKO).

Statistical analysis. Unpaired ANOVA with Tukey-Kramer post-hoc test and Wilcoxon's signed rank test were used for comparisons between groups. Results are shown as the mean \pm S.D. The statistical significance is indicated as follows: $* * p<0.01$ and $* p<0.05$.

\section{Results}

GGOH does not significantly affect skeletal muscle regeneration. There are no reports in the literature describing oral administration of GGOH in vivo. Therefore, we first optimized dosage parameters to minimize adverse effects. Our initial dose of $3,000 \mathrm{mg} / \mathrm{ml} / \mathrm{kg} /$ day led to a reduction in body weight and food intake (Data not shown). After careful dose titration, we eventually found that the administration of $300 \mathrm{mg} / \mathrm{kg} /$ day with a rest day every fourth day (Figure 1A) did not affect body weight (Figure 1B) or food intake (Figure 1C). This dosage regimen was then used for all further experiments described.

To examine the effect of $\mathrm{GGOH}$ on skeletal muscle regeneration in vivo, we first created a Cardiotoxin (CTX) injury muscle regeneration model. Intraoral administration of GGOH did not affect total body (Figure 2A) or tibial anterior (TA) muscle weight following CTX injection and muscle regeneration (Figure $2 \mathrm{~B}$ ). GGOH did not significantly increase the size of regenerated fibers with central nuclei (Figure 2C and D). We also examined the expression of myogenesis marker genes such as Myogenin (Figure 2E) and Myosin heavy chain (Myhc1) (Figure 2F). However, GGOH intake did not affect Myogenin expression although Myhc1 was slightly 

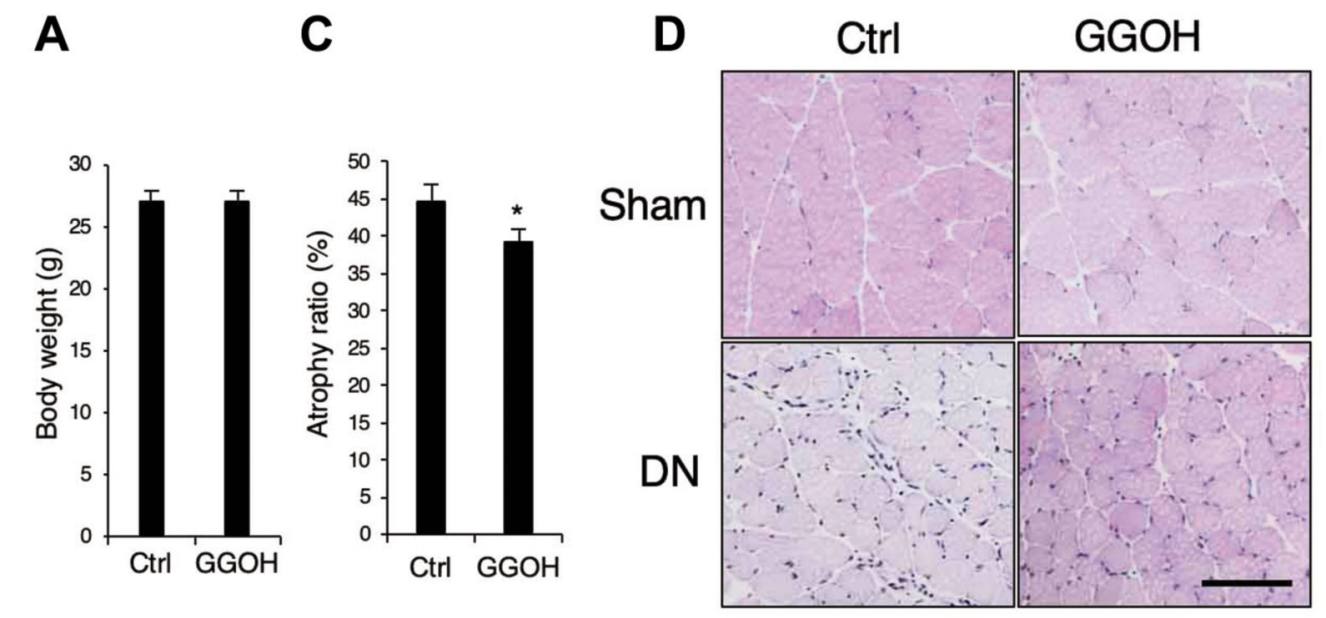

$\mathbf{E}$
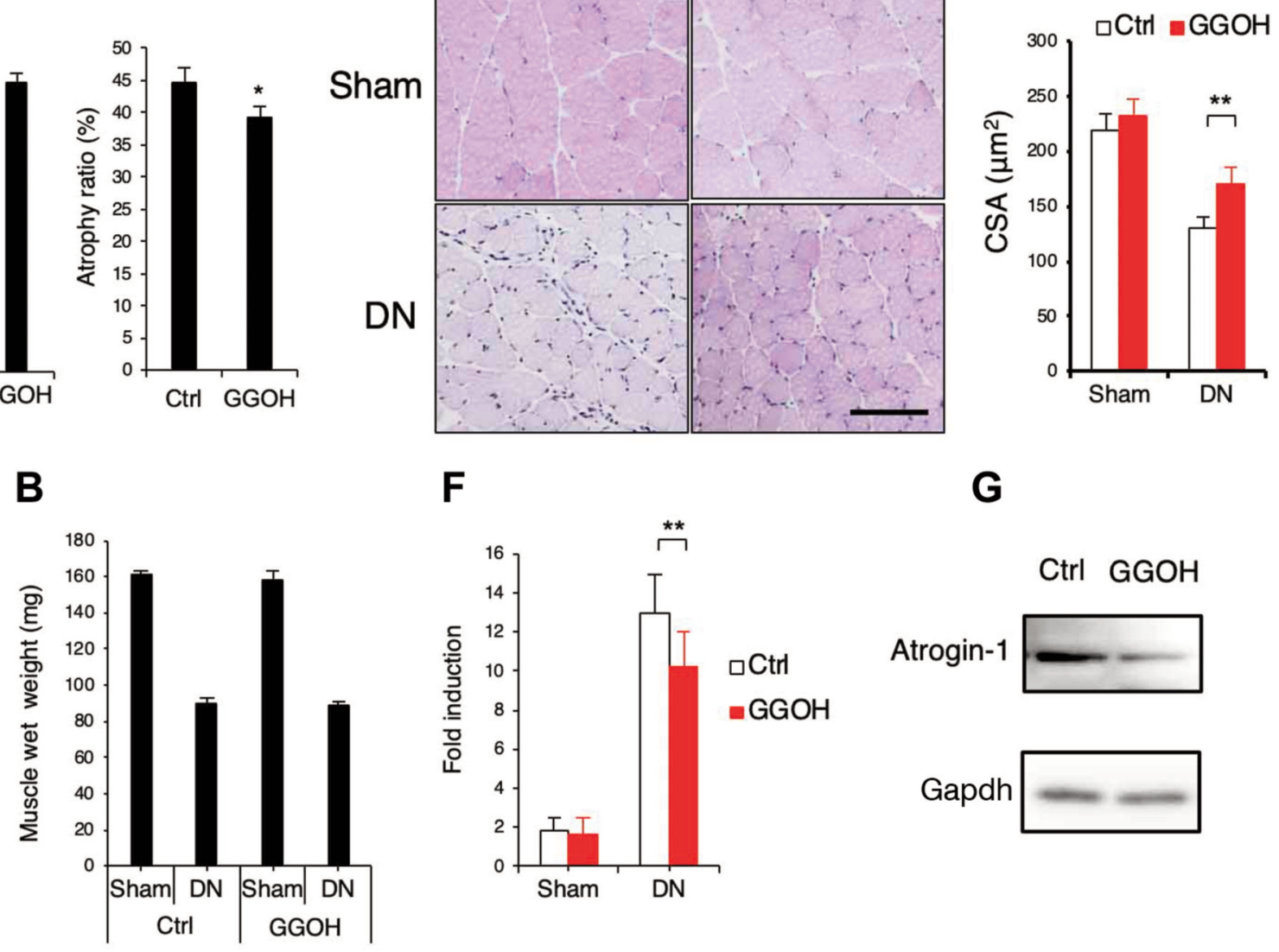

G

\section{Ctrl GGOH}

Atrogin-1
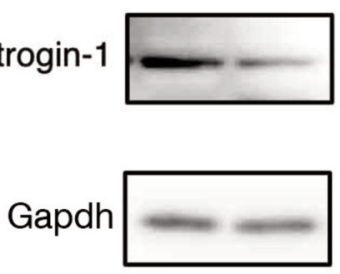

Figure 3. Administration of GGOH prevents muscle atrophy by denervation. (A-C) The effects of oral administration of GGOH on total body weight (A), and wet weight of gastrocnemius muscles $(G A)(B)$. Atrophy ratio (\%): (sham side muscle wet weight $-D N$ side muscle wet)/sham side muscle wet $\times 100(C)$, or muscle cross sectional area (CSA). Scale $=100 \mu \mathrm{m}(D)$. CSA was quantified $(E)$. (F and $G)$ The mRNA $(F)$ or protein $(G)$ levels of Atrogin-1 from the GA muscle with or without GGOH administration were determined by quantitative real time PCR $(F)$ or western blotting $(G)$ respectively. Representative images are shown $(D$ and $G)$. Data are mean $\pm S D(n=10)$. ${ }^{*} p<0.01,{ }^{*} p<0.05$, versus control (Ctrl).

increased. Thus, our data suggests that GGOH administration does not enhance skeletal muscle regeneration in vivo.

\section{Administration of GGOH prevents muscle atrophy induced by denervation. We next examined whether intraoral administration of $\mathrm{GGOH}$ affects the atrophy of skeletal muscle in vivo. Intraoral administration of GGOH did not affect total body weight (Figure 3A) or wet weight of intact or denervated GA muscle (Figure 3B). However, GGOH reduced the atrophy ratio of the GA muscle (Figure 3C) and the decrease in cross-sectional area of muscle fibers (Figure 3D and E). Administration of GGOH also suppressed the expression levels of Atrogin-1 in denervation-induced muscle atrophy (Figure 3F and G).}

The effect of GGOH on DEX-induced muscle atrophy in vitro. Finally, we examined the effect of GGOH on Atrogin-
1 expression and muscle atrophy in vitro. We used a common well-characterized dexamethasone-induced atrophy model of $\mathrm{C} 2 \mathrm{C} 12$ cells based on glucocorticoid skeletal muscle atrophy. Sandri M et al., (2004) demonstrated that treatment of C2C12 myoblast with glucocorticoid activated the atrophy related genes expression and increased the protein degradation (15). GGOH treatment firmly suppressed expression of Atrogin-1 (Figure 4A and B) and the decrease of skeletal muscle fiber size (Figure $4 \mathrm{C}$ and D) in a dosedependent manner.

\section{Discussion}

In this study, the effect of GGOH administration was limited to an increase in fiber size during denervation-induced atrophy in vivo. Consistent with this, GGOH has been previously shown to enhance the differentiation of $\mathrm{C} 2 \mathrm{C} 12$ 
A

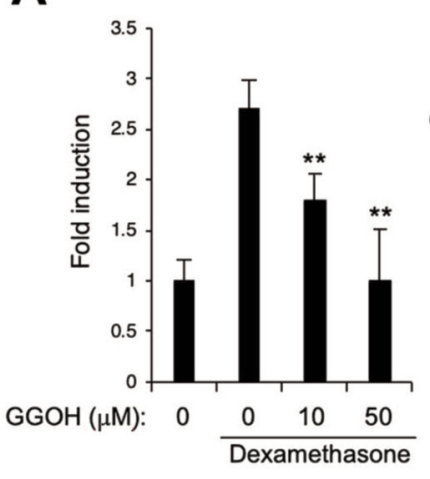

B

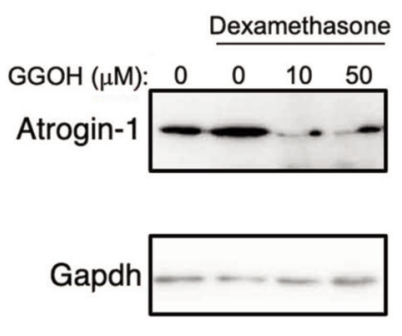

C

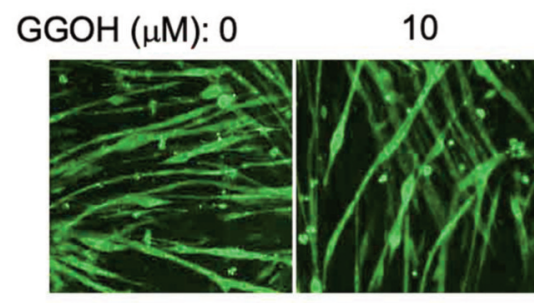

50

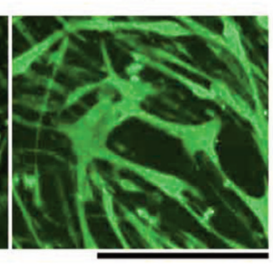

D

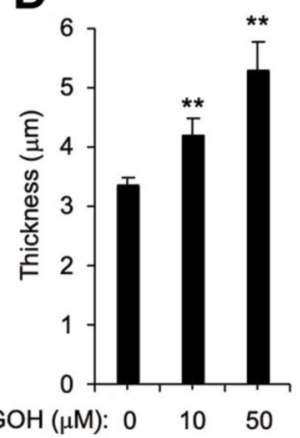

Figure 4. The effect of GGOH on DEX-induced muscle atrophy in vitro. C2C12 cells were differentiated by treatment with myogenic medium for 6 days after which cells were cultured in the presence of $1 \mu \mathrm{M}$ dexamethasone along with 0,10 , or $50 \mu M$ GGOH for 2 days. $m R N A$ (A) or protein (B) levels of Atrogin-1 were assessed by quantitative real time PCR (A) or Western blotting method (B), respectively. Myosin heavy chain positive myotubes were determined by immunocytochemistry. Representative images are shown. Scale $=500 \mu m(C)$. The width of each fiber was quantified (D). Data are mean $\pm S D(n=4)$. ** $p<0.01$, versus control (Ctrl) or DMSO treatment.

myoblasts in vitro (12). Our previous report also showed that high doses of GGOH tend to suppress proliferation of myoblasts (12). In the process of skeletal muscle regeneration, myoblast differentiation occurs following the proliferation of myoblasts. Therefore, GGOH may negatively affect proliferation during the early stage of regeneration thereby neutralizing any positive enhancement gained during its effects on differentiation at the late stage of regeneration.

Atrogin-1 expression is increased during muscle atrophy induced by a variety of stressors including fasting, denervation, glucocorticoids, cachexia, or statins (17). Atrogin-1, as an E3 ubiquitin ligase, is thought to cause degradation of skeletal muscle proteins via ubiquitination. Thus, control of Atrogin-1 expression is important for establishing a method to prevent skeletal muscle atrophy. Cao et al., have reported that $\mathrm{GGOH}$ reduces statin-induced protein levels of Atrogin-1 by stimulating geranylgeranylation (11). In our study, GGOH also suppressed the expression of denervation-induced or glucocorticoid-induced Atrogin-1.
Numerous studies have demonstrated a role of NF-kB in the induction of muscle atrophy under conditions such as disuse $(18,19)$, denervation (20), aging (21), and cachexia $(22,23)$. Muscle atrophy induced by elevated NF-kB was found to be associated with increased expression of Atrogin1 , and NF-kB directly regulates transcription of Atrogin-1 (24). Feeding or treatment with GGOH has been shown to reduce NF-KB signaling induced by lipopolysaccharide in the rat liver or in human macrophage-like THP-1 cells $(25,26)$. Furthermore, GGOH has also been shown to stimulate the production of testosterone in testis-derived cells (27). Testosterone is the main steroid hormone secreted by the testes and is strongly involved in the process of growth, maintenance and repair of muscle mass (28). Therefore, NF$\mathrm{KB}$ signaling and/or testosterone may be involved in the inhibitory effect of $\mathrm{GGOH}$ on denervation induced muscle atrophy. Needless to mention, additional experiments are required to elucidate the exact effect of GGOH on skeletal muscle metabolism. 
In this study, we demonstrated that GGOH rescues skeletal muscle atrophy in vivo. Since GGOH is inexpensive, categorized as GRAS and can be taken orally (25) our data may lead to the development of novel treatments for muscle atrophies including sarcopenia.

\section{Funding}

This study has been supported by Suzuken Memorial Foundation and Japan Health Foundation.

\section{Conflicts of Interest}

The Authors declare that they have no conflicts of interest regarding this study. Y.A. is an employee of Mitsubishi-Chemical Foods Corporation.

\section{Authors' Contributions}

A.M., T.R, S.H., M.U., T.M., and S.K. performed the experiments. A.M., T.R, S.H., Y.A., M.U., A.I., T.M., K.M., K.T., and S.K. reviewed the intermediate draft. S.K. designed the study. S.K. performed the literature review, prepared the initial and final versions of the article, and submitted the document.

\section{Acknowledgements}

The Authors are grateful to William N. Addison (Kyushu Dental University) for the comments and assistance during this work.

\section{References}

1 Delmonico MJ, Harris TB, Lee JS, Visser M, Nevitt M, Kritchevsky SB, Tylavsky FA, Newman AB, Health A and Body Composition S: Alternative definitions of sarcopenia, lower extremity performance, and functional impairment with aging in older men and women. J Am Geriatr Soc 55(5): 769-774, 2007. PMID: 17493199. DOI: 10.1111/j.1532-5415.2007.01140.x

2 Woo J: Sarcopenia. Clin Geriatr Med 33(3): 305-314, 2017. PMID: 28689564. DOI: 10.1016/j.cger.2017.02.003

3 Mitch WE and Goldberg AL: Mechanisms of muscle wasting. The role of the ubiquitin-proteasome pathway. N Engl J Med 335(25): 1897-1905, 1996. PMID: 8948566. DOI: $10.1056 /$ NEJM199612193352507

4 Attaix D, Mosoni L, Dardevet D, Combaret L, Mirand PP and Grizard J: Altered responses in skeletal muscle protein turnover during aging in anabolic and catabolic periods. Int J Biochem Cell Biol 37(10): 1962-1973, 2005. PMID: 15905114. DOI: 10.1016/j.biocel.2005.04.009

5 Gomes MD, Lecker SH, Jagoe RT, Navon A and Goldberg AL: Atrogin-1, a muscle-specific f-box protein highly expressed during muscle atrophy. Proc Natl Acad Sci U S A 98(25): 1444014445, 2001. PMID: 11717410. DOI: 10.1073/pnas.251541198

6 Bodine SC, Latres E, Baumhueter S, Lai VK, Nunez L, Clarke BA, Poueymirou WT, Panaro FJ, Na E, Dharmarajan K, Pan ZQ, Valenzuela DM, DeChiara TM, Stitt TN, Yancopoulos GD and Glass DJ: Identification of ubiquitin ligases required for skeletal muscle atrophy. Science 294(5547): 1704-1708, 2001. PMID: 11679633. DOI: $10.1126 /$ science. 1065874

7 Muraguchi T, Okamoto K, Mitake M, Ogawa H and Shidoji Y: Polished rice as natural sources of cancer-preventing geranylgeranoic acid. J Clin Biochem Nutr 49(1): 8-15, 2011. PMID: 21765600. DOI: 10.3164/jcbn.10-110

8 Frenkel J, Rijkers GT, Mandey SH, Buurman SW, Houten SM, Wanders RJ, Waterham HR and Kuis W: Lack of isoprenoid products raises ex vivo interleukin-1beta secretion in hyperimmunoglobulinemia $\mathrm{d}$ and periodic fever syndrome. Arthritis Rheum 46(10): 2794-2803, 2002. PMID: 12384940. DOI: $10.1002 /$ art. 10550

9 Walker K and Olson MF: Targeting ras and rho gtpases as opportunities for cancer therapeutics. Curr Opin Genet Dev 15(1): 62-68, 2005. PMID: 15661535. DOI: 10.1016/j.gde. 2004.11.001

10 Thompson PD, Clarkson P and Karas RH: Statin-associated myopathy. JAMA 289(13): 1681-1690, 2003. PMID: 12672737. DOI: $10.1001 /$ jama.289.13.1681

11 Cao P, Hanai J, Tanksale P, Imamura S, Sukhatme VP and Lecker SH: Statin-induced muscle damage and atrogin-1 induction is the result of a geranylgeranylation defect. FASEB J 23(9): 28442854, 2009. PMID: 19406843. DOI: 10.1096/fj.08-128843

12 Matsubara T, Urata M, Nakajima T, Fukuzaki M, Masuda R, Yoshimoto Y, Addison WN, Nakatomi C, Morikawa K, Zhang M, Saeki K, Takahashi Y, Nakamichi A and Kokabu S: Geranylgeraniol-induced myogenic differentiation of c2c12 cells. In Vivo 32(6): 1427-1431, 2018. PMID: 30348697. DOI: 10.21873/invivo.11395

13 Abe T, Kohno S, Yama T, Ochi A, Suto T, Hirasaka K, Ohno A, Teshima-Kondo S, Okumura Y, Oarada M, Choi I, Mukai R, Terao $\mathrm{J}$ and Nikawa T: Soy glycinin contains a functional inhibitory sequence against muscle-atrophy-associated ubiquitin ligase cbl-b. Int J Endocrinol 2013: 907565, 2013. PMID: 23762056. DOI: 10.1155/2013/907565

14 Kokabu S, Nakatomi C, Matsubara T, Ono Y, Addison WN, Lowery JW, Urata M, Hudnall AM, Hitomi S, Nakatomi M, Sato T, Osawa K, Yoda T, Rosen V and Jimi E: The transcriptional co-repressor tle3 regulates myogenic differentiation by repressing the activity of the myod transcription factor. J Biol Chem 292(31): 12885-12894, 2017. PMID: 28607151. DOI: 10.1074/jbc.M116.774570

15 Sandri M, Sandri C, Gilbert A, Skurk C, Calabria E, Picard A, Walsh K, Schiaffino S, Lecker SH and Goldberg AL: Foxo transcription factors induce the atrophy-related ubiquitin ligase atrogin-1 and cause skeletal muscle atrophy. Cell 117(3): 399-412, 2004. PMID: 15109499. DOI: 10.1016/s0092-8674(04)00400-3

16 Livak KJ and Schmittgen TD: Analysis of relative gene expression data using real-time quantitative pcr and the 2(-delta delta $\mathrm{c}(\mathrm{t}))$ method. Methods 25(4): 402-408, 2001. PMID: 11846609. DOI: $10.1006 /$ meth.2001.1262

17 Bodine SC and Baehr LM: Skeletal muscle atrophy and the E3 ubiquitin ligases murf1 and mafbx/atrogin-1. Am J Physiol Endocrinol Metab 307(6): E469-484, 2014. PMID: 25096180. DOI: $10.1152 /$ ajpendo.00204.2014

18 Judge AR, Koncarevic A, Hunter RB, Liou HC, Jackman RW and Kandarian SC: Role for ikappabalpha, but not c-rel, in skeletal muscle atrophy. Am J Physiol Cell Physiol 292(1): C372-382, 2007. PMID: 16928772. DOI: 10.1152/ajpcell.00293. 2006 
19 Van Gammeren D, Damrauer JS, Jackman RW and Kandarian SC: The ikappab kinases ikkalpha and ikkbeta are necessary and sufficient for skeletal muscle atrophy. FASEB J 23(2): 362-370, 2009. PMID: 18827022. DOI: 10.1096/fj.08-114249

20 Mourkioti F, Kratsios P, Luedde T, Song YH, Delafontaine P, Adami R, Parente V, Bottinelli R, Pasparakis M and Rosenthal $\mathrm{N}$ : Targeted ablation of ikk2 improves skeletal muscle strength, maintains mass, and promotes regeneration. J Clin Invest 116(11): 2945-2954, 2006. PMID: 17080195. DOI: 10.1172/ JCI28721

21 Bar-Shai M, Carmeli E, Ljubuncic P and Reznick AZ: Exercise and immobilization in aging animals: The involvement of oxidative stress and nf-kappab activation. Free Radic Biol Med 44(2): 202-214, 2008. PMID: 18191756. DOI: 10.1016/ j.freeradbiomed.2007.03.019

22 Cai D, Frantz JD, Tawa NE, Jr., Melendez PA, Oh BC, Lidov HG, Hasselgren PO, Frontera WR, Lee J, Glass DJ and Shoelson SE: Ikkbeta/nf-kappab activation causes severe muscle wasting in mice. Cell 119(2): 285-298, 2004. PMID: 15479644. DOI: 10.1016/j.cell.2004.09.027

23 Rhoads MG, Kandarian SC, Pacelli F, Doglietto GB and Bossola $\mathrm{M}$ : Expression of nf-kappab and ikappab proteins in skeletal muscle of gastric cancer patients. Eur J Cancer 46(1): 191-197, 2010. PMID: 19857958. DOI: 10.1016/j.ejca.2009.10.008

$24 \mathrm{Wu}$ CL, Kandarian SC and Jackman RW: Identification of genes that elicit disuse muscle atrophy via the transcription factors p50 and bcl-3. PLoS One 6(1): e16171, 2011. PMID: 21249144. DOI: $10.1371 /$ journal.pone.0016171
25 Giriwono PE, Shirakawa H, Ohsaki Y, Hata S, Kuriyama H, Sato $\mathrm{S}$, Goto $\mathrm{T}$ and Komai $\mathrm{M}$ : Dietary supplementation with geranylgeraniol suppresses lipopolysaccharide-induced inflammation via inhibition of nuclear factor-kappab activation in rats. Eur J Nutr 52(3): 1191-1199, 2013. PMID: 22847643. DOI: $10.1007 / \mathrm{s} 00394-012-0429-y$

26 Giriwono PE, Shirakawa H, Ohsaki Y, Sato S, Aoyama Y, Ho HJ, Goto $\mathrm{T}$ and Komai M: Geranylgeraniol suppresses the expression of irak1 and traf6 to inhibit nfkappab activation in lipopolysaccharide-induced inflammatory responses in human macrophage-like cells. Int J Mol Sci 20(9): 2320, 2019. PMID: 31083375. DOI: 10.3390/ijms20092320

27 Ho HJ, Shirakawa H, Yoshida R, Ito A, Maeda M, Goto T and Komai M: Geranylgeraniol enhances testosterone production via the camp/protein kinase a pathway in testis-derived i-10 tumor cells. Biosci Biotechnol Biochem 80(4): 791-797, 2016. PMID: 26757775. DOI: 10.1080/09168451.2015.1123612

28 Carson JA and Manolagas SC: Effects of sex steroids on bones and muscles: Similarities, parallels, and putative interactions in health and disease. Bone 80: 67-78, 2015. PMID: 26453497. DOI: $10.1016 /$ j.bone.2015.04.015

Received May 19, 2020

Revised June 4, 2020

Accepted June 5, 2020 